\title{
Photonic Microresonators Created by Slow Optical Cooking
}

\author{
Gabriella Gardosi,* Brian J. Mangan, Gabe S. Puc, and Michael Sumetsky
}

Cite This: ACS Photonics 2021, 8, 436-442

Read Online

ABSTRACT: Silica and water are known as exceptionally inert chemical materials whose interaction is not completely understood. Here we show that the effect of this interaction can be significantly enhanced by optical whispering gallery modes (WGMs) propagating in a silica microcapillary filled with water. Our experiments demonstrate that WGMs, which evanescently heat liquid water over several hours, induce permanent alterations in silica material characterized by the subnanometer variation of the WGM spectrum. We use the discovered effect to fabricate optical WGM microresonators having potential applications in optical signal processing and microfluidic sensing. Our results pave the way for

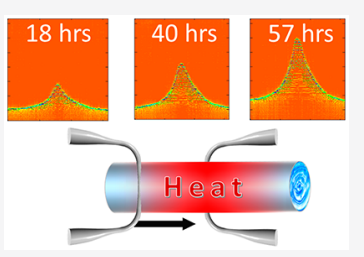
the ultraprecise fabrication of resonant optical microdevices and the ultra-accurate characterization of physical and chemical processes at solid-liquid interfaces.

KEYWORDS: surface nanoscale photonics, silica-water interface, whispering gallery modes, optical microresonators

$S^{\text {into }}$ ilica is one of the most ubiquitous materials whose applications in modern technology and societal life range from optical fibers in telecommunications to glass containers in the food industry. The broad use of silica arises in part from its chemical inertness at normal temperature and pressure and much beyond these conditions. The latter determines the lifetime, reliability, and safety of silica devices, apparatus, and common items fabricated from silica. One of the important properties of silica is its inertness to water. The silica-water reaction has been a matter of intensive multidisciplinary research over the last decades (see refs $1-7$ and references therein). The silica-water interaction is critical for the understanding of geochemical and environmental processes, ${ }^{3,8,9}$ silica in drinking water effects, ${ }^{10}$ biocomposite desilicification mechanisms, ${ }^{2}$ and safe radioactive waste containment, ${ }^{11,12}$ as well as for fundamental studies of solidliquid interfaces. ${ }^{5-7}$ The research challenge lies in detecting very slow and complex processes, including both chemical and physical transformations at and near the silica-water interface, which are not completely understood and remain controversial (see, e.g., refs 3-9, and 13).

Developed experimental methods to investigate the silicawater interactions include vibrational spectroscopy, $^{5,7,14-19,35,36}$ calorimetry, $^{20,21} \mathrm{STM}^{22}$ AFM, ${ }^{24,30}$ TEM, $^{25}$ optical fiber transmission of light, ${ }^{26}$ inelastic deformation of optical fibers, ${ }^{27-29}$ and others. These methods have demonstrated hydration and hydrolysis, ${ }^{14-25}$ structural and stress relaxation, ${ }^{14,27-29}$ crack formation, ${ }^{30,31}$ volume expansion, ${ }^{4}$ deep water diffusion, ${ }^{26}$ and silica dissolution. ${ }^{32-37}$ Due to the inertness of the silica-water interaction, temporal variations caused by processes near the silica-water interface have been required to last over days and sometimes years to be measurable. ${ }^{1,2,4,11-14,22,24,26,29,31-37}$ Previous work accelerated these processes by using high temperature and pressure ${ }^{14,33-35}$ or by increasing the surface area of the reaction using silica microparticles ${ }^{32,36}$ or porous silica. ${ }^{18,37}$ The insight into the physics and chemistry of these processes at environmental temperatures and pressures, which are most important for applications, requires the development of approaches enabling exceptional temporal and spatial resolution of alterations near the silica-water interface.

In this Letter, we show that it is possible to initiate, enhance, and characterize the dramatically small alterations near the interface of silica and liquid water with optical whispering gallery modes (WGMs) excited in a water-filled silica microcapillary fiber (MCF). Optical WGM microresonators and WGM microcapillaries in particular have found numerous applications ranging from ultraprecise microfluidic and microparticle sensing ${ }^{38-41}$ to signal processing in telecommunication, frequency comb generation, and quantum networking. ${ }^{38,42-44}$ Our experiments demonstrate that the WGMs excited in a silica microcapillary filled with water can induce temporal as well as permanent alterations of the silica material and perform an unprecedentedly precise characterization of these alterations with the minute-scale temporal resolution, micron-scale spatial resolution along the MCF length, and picometer resolution in WGM spectrum variation. Simultaneously, we suggest a new ultraprecise method for fabrication of WGM microresonator devices (also called SNAP devices ${ }^{45,46}$ ), which promise a range of important applications in optical signal processing and microfluidic sensing. Since our fabrication method involves hot water, which irreversibly

Received: December 7, 2020

Published: January 14, 2021 
interacted with silica over multiple hours, we have coined this technique as the slow cooking of optical microresonators.

Our experimental setup illustrated in Figure 1, consisted of a silica MCF, drawn from a silica preform at OFS Laboratories,

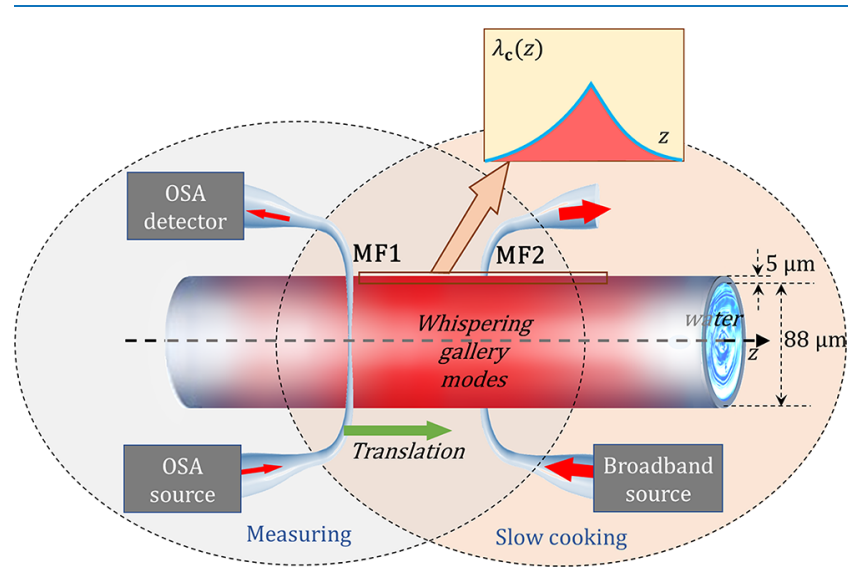

Figure 1. Experimental setup. A silica MCF filled with water was coupled to two microfibers, MF1 and MF2, which were oriented perpendicular to the MCF. MF1 was connected to the OSA, which measured the cutoff wavelength profile $\lambda_{c}(z)$ along the MCF, shown in the inset. The broadband light was coupled into the MCF via MF2, which was maintained at a fixed position. The heating effect along the MCF caused by the broadband WGMs is illustrated in red.

and two input-output U-shaped biconical tapers with microndiameter waists, fabricated by pulling a commercial single mode fiber in a ceramic heater. We first prepared a short length $(<15 \mathrm{~cm})$ of MCF by chemical etching to remove its external polymer coating and obtain bare silica. A middle section of 3 $\mathrm{cm}$ was submerged in $\mathrm{H}_{2} \mathrm{SO}_{4}$ for $2 \mathrm{~min}$ at $\sim 120{ }^{\circ} \mathrm{C}$ and then rinsed in deionized water. The outer MCF radius $r_{0}=45 \mu \mathrm{m}$ and wall thickness of $5 \mu \mathrm{m}$ were measured using an optical microscope. After mounting the MCF onto an aluminum holder, we connected one end to a syringe of deionized water and kept the other end open. The waists of the microfibers MF1 and MF2 were oriented normally to the MCF. We connected MF1 to the optical spectrum analyzer (OSA) with spectral resolution $1.3 \mathrm{pm}$ in the bandwidth $1520-1610 \mathrm{~nm}$ (Luna OVA5000). The measuring MF1 was translated along the MCF, periodically contacting it with the required spatial resolution. At these points, light that coupled from MF1 into the MCF excited WGMs, which contributed to the transmission power spectrogram $P(\lambda, z)$ measured as a function of wavelength $\lambda$ and coordinate $z$ along the MCF axis. Generally, the transmission power obtained experimentally includes the effects of light propagation inside the MCF and outside it (e.g., along the microfiber taper). To determine the $P(\lambda, z)$ of the MCF under study, we treated the measured transmission power, as explained in Supporting Information, Section 1. We connected the heating MF2 to the broadband light source (1530-1610 nm) amplified by an erbium-doped fiber amplifier transmitting the output optical power tunable up to $100 \mathrm{~mW}$. MF2 was positioned in direct contact to the MCF. Light coupled from MF2 into the MCF-excited WGMs, which evanescently penetrated into the MCF and were partly absorbed by water, causing heating, ${ }^{47}$ water motion, ${ }^{48}$ and other reversible and irreversible physical and chemical changes of the MCF wall and surfaces.
Theoretically, the WGMs in the MCF, whose characteristics (e.g., internal and external radii and refractive index) experience small variations along the $z$ axis, can be determined by the separation of variables in cylindrical coordinates. These modes are numerated by the azimuthal and radial quantum numbers, $m$ and $p$, and polarization, $s .{ }^{49}$ As both MF1 and MF2 in Figure 1 were oriented normally to the MCF, the excited WGMs were primarily directed tangentially to the MCF axis. For this reason, the excited modes propagate slowly along the MCF with a small propagation constant $\beta_{c}(\lambda, z)$ that vanishes at the cutoff wavelengths (CWs) $\lambda=\lambda_{c}(z) .{ }^{45-47}$ Here, for briefness, we use notation $c=(m, p, s)$. Within the MCF spectrograms $P(\lambda, z)$, the cutoff wavelengths $\lambda_{c}(z)$ correspond to the narrow transmission power dips that characterize the fiber's nonuniformity (see, e.g., ref 47). For an ideally uniform fiber, the CWs are independent of $z$. For a fiber with small nonuniformities, the propagation constant of slow WGMs is found to be $\beta_{c}(\lambda, z)=2^{3 / 2} \pi n_{\mathrm{r}} \lambda_{c}^{-3 / 2}\left(\lambda_{c}(z)+i \gamma_{c}-\lambda\right)^{1 / 2}$, where $n_{\mathrm{r}}$ is the effective refractive index of the fiber and $\gamma_{c}$ determines its material losses. Thus, for slow WGMs, that is, when $\lambda$ is close to $\lambda_{c}(z)$, the propagation constant $\beta_{c}(\lambda, z)$ and, consequently, the transmission power $P(\lambda, z)$ are sensitive to very small nonuniformities of the refractive index and cross-section of the MCF characterized by the CW profile $\lambda_{c}(z)$.

Our slow cooking experiments are represented in Figure 2. First, we characterized the nonuniformity of an original $5 \mathrm{~mm}$ long MCF segment filled with water by measuring its spectrogram, shown in Figure 2a. To this end, MF2 in Figure 1 was disconnected from the MCF. MF1 was then translated along the MCF periodically making contact at points $z_{j}$ where the transmission power spectrum $P\left(\lambda, z_{j}\right)$ was measured with spatial resolution $\Delta z=z_{j+1}-z_{j}=20 \mu \mathrm{m}$.

Transmission power dips (green-blue lines) determine the CW profiles $\lambda_{c}(z)$. In this spectrogram we observe similar CWs corresponding to the azimuthal quantum numbers $m$ different by unity. The MCF external radius can be determined from this spectrogram as $r_{0}=\left(\lambda_{c}^{2} /\left(2 \pi n_{\mathrm{r}} \Delta \lambda_{c}^{\mathrm{FSR}}\right)\right)$, where $\Delta \lambda_{c}^{\mathrm{FSR}}=$ $\left|\lambda_{m+1, p, s}-\lambda_{m, p, s}\right|$ is the azimuthal free spectral range, labeled with a black arrow on Figure 2a1. For our silica MCF, setting $n_{\mathrm{r}}=1.44, \lambda_{c}=1548.4 \mathrm{~nm}$, and $\Delta \lambda_{c}^{\mathrm{FSR}}=5.9 \mathrm{~nm}$, we find $r_{0}=45$ $\mu \mathrm{m}$ is in good agreement with the radius value obtained with an optical microscope. The nonuniformity $\Delta r$ of the $5 \mathrm{~mm}$ long MCF segment considered was determined from the CW variation $\Delta \lambda \sim 0.1 \mathrm{~nm}$ found from the Figure 2a1, which magnifies the blue box region in Figure 2a near $\lambda_{c}=1548 \mathrm{~nm}$. From the rescaling relation $\Delta r / r_{0}=\Delta \lambda / \lambda_{c}$ (see, e.g., ref 45) we found the nanoscale nonuniformity of this segment $\Delta r \sim 3 \mathrm{~nm}$.

Having demonstrated sufficiently small nonuniformities of the MCF, we next investigated the $\mathrm{CW}$ variation during its heating with light coupled from MF2. We placed MF2 carrying the broadband light of $63 \mathrm{~mW}$ power in contact with the center of the same water-filled MCF segment. This power was more than 2 orders of magnitude greater than the $0.2 \mathrm{~mW}$ power launched from the OSA into the sensing MF1. Therefore, the effect of WGMs excited by MF1 can be ignored. The evanescent tails of WGMs excited by this microfiber (as well as small fraction of light experiencing direct refraction) penetrated into water and caused its heating ${ }^{47}$ and motion. ${ }^{48}$ The spectrogram spatial resolution was $\Delta z=10 \mu \mathrm{m}$, measured over $80 \mathrm{~min}$. Figure $2 \mathrm{~b} 1$ magnifies the blue box in Figure $2 \mathrm{~b}$ and clearly demonstrates the creation of a temporary bottle microresonator. The formation of a temporary resonator is similar to the local heating of an optical fiber with a $\mathrm{CO}_{2}$ 


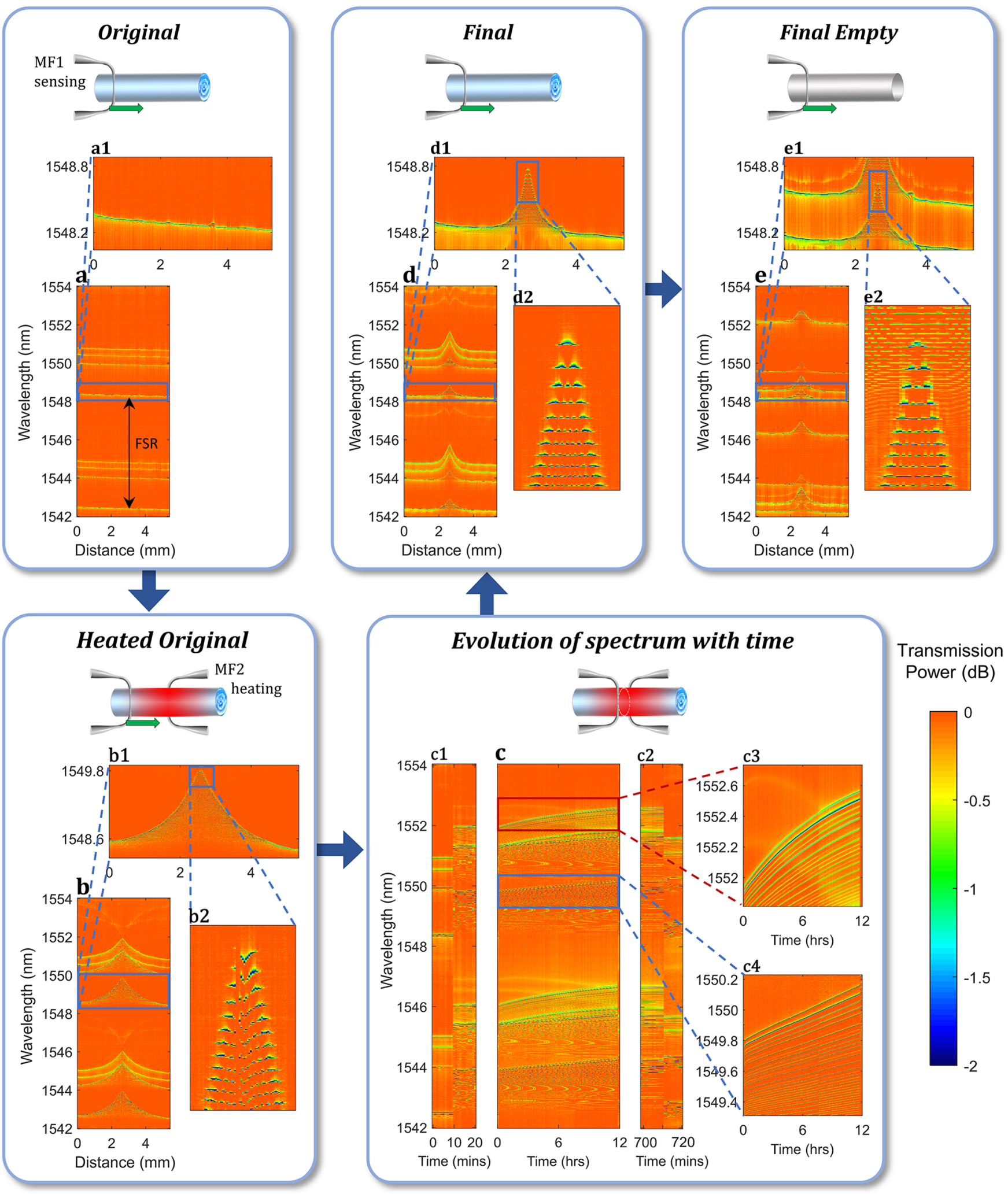

Figure 2. Slow cooking process. (a) The control spectrogram, which determines the original cutoff wavelength variation. (b) The spectrogram of the temporary resonator formed by heating with magnified parts shown in (b1) and (b2). (c) The temporal spectrogram showing the variation of spectrum as a function of time at the position of MF2 during the process of slow cooking with magnified parts shown in (c1) - (c4). (d) The spectrogram showing the formation of a permanent microresonator, with magnified parts shown in (d1) and (d2). (e) The spectrogram of the same permanent resonator after water is removed from the MCF and dried for $12 \mathrm{~h}$. (e1) and (e2) are magnified parts of spectrogram (e).

laser $^{50}$ and a MCF with an inserted metal wire. ${ }^{51}$ However, in Supporting Information, Section 2, we show that heating is caused by WGMs distributed along $4 \mathrm{~mm}$ of the MCF rather than a heat source localized at the MF2 contact position. 

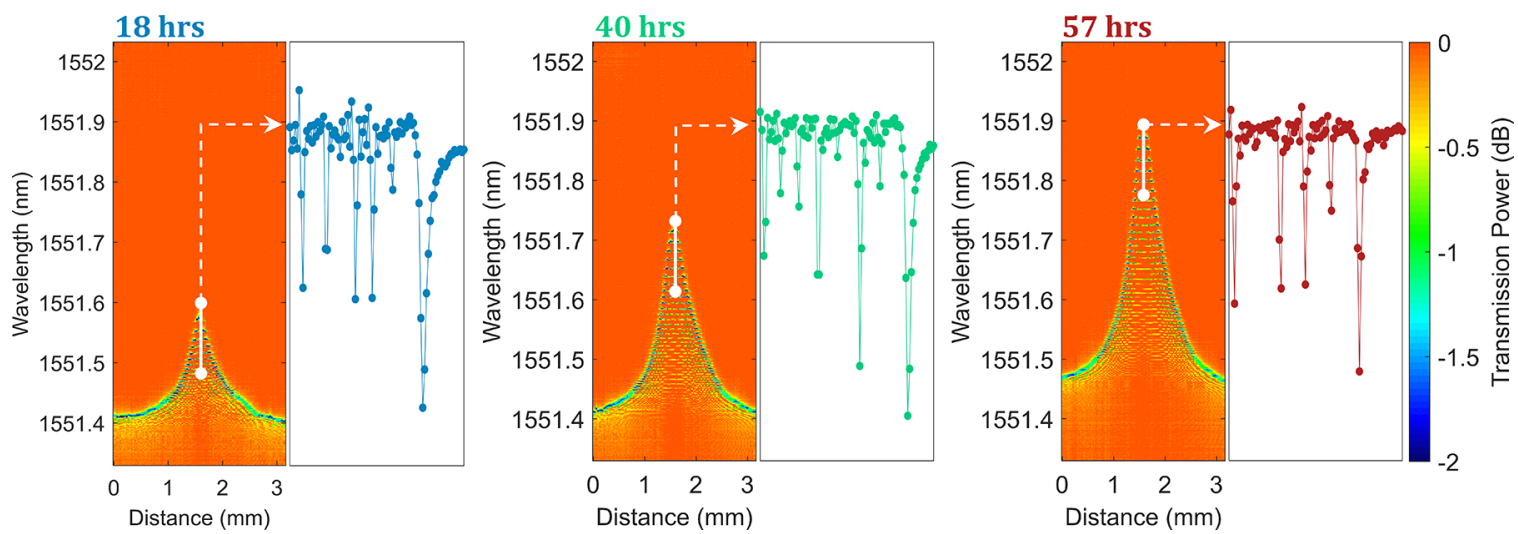

Figure 3. Spectrograms of permanently induced microresonators cooked over different time periods. $(\mathrm{a}-\mathrm{c})$ Left: spectrograms of the same MCF after slow cooking for total duration of 18,40 , and $57 \mathrm{~h}$. Right: spectrum at the top CWs (labeled in white) of the corresponding spectrogram.

Further magnification in Figure $2 \mathrm{~b} 2$ shows that the axial eigenwavelengths decrease near the MF2 position instead of remaining constant along the whole resonator length. We suggest that this decrease is a measurement artifact caused by power leakage through MF1.

After $80 \mathrm{~min}$, heating was turned off and we observed no perceptible changes of CW. Therefore, the bottle microresonator shown in Figure $2 \mathrm{~b}$ is primarily induced by the reversible refractive index variation of the silica and water, $n_{\mathrm{s}}$ and $n_{\mathrm{w}}$, with temperature $T$. Indeed, for silica and water, we have $\frac{1}{n_{\mathrm{s}}} \frac{\mathrm{d} n_{\mathrm{s}}}{\mathrm{d} T}=8 \times 10^{-6} /{ }^{\circ} \mathrm{C}$ and $\frac{1}{n_{\mathrm{w}}} \frac{\mathrm{d} n_{\mathrm{w}}}{\mathrm{d} T}=-7 \times 10^{-5} /{ }^{\circ} \mathrm{C}$, while the corresponding relative expansion of the fiber radius is $\frac{1}{r_{0}} \frac{\mathrm{d} r_{0}}{\mathrm{~d} T}=0.6 \times 10^{-6} /{ }^{\circ} \mathrm{C}$. Assuming that the temperature distribution over the MCF cross-section is uniform, we can estimate its variation along the MCF length as

$$
\Delta T(z)=\left(\frac{F_{c}^{(s)}}{n_{\mathrm{s}}} \frac{\mathrm{d} n_{\mathrm{s}}}{\mathrm{d} T}+\frac{F_{c}^{(\mathrm{w})}}{n_{\mathrm{w}}} \frac{\mathrm{d} n_{\mathrm{w}}}{\mathrm{d} T}+\frac{1}{r_{0}} \frac{\mathrm{d} r_{0}}{\mathrm{~d} T}\right)^{-1} \frac{\Delta \lambda_{c}(z)}{\lambda_{c}}
$$

where $F_{c}^{(s)}$ and $F_{c}^{(\mathrm{w})}=1-F_{c}^{(\mathrm{s})}$ are the fractions of a WGM with quantum numbers $c=(m, p, s)$, which are localized in the capillary wall and water, respectively. The CW variation $\Delta \lambda_{c}(z)$ in eq 1 vanishes away from the MF2 position. We assume that the $\mathrm{CW}$ variation shown in Figure $2 \mathrm{bl}$ corresponds to the WGM localized primarily in the silica wall, that is, $F_{c}^{(s)}=1$ and $F_{c}^{(w)}=0$. We measured the CW heating shift at the MF2 position $z=z_{\mathrm{MF} 2}$, equal to $\Delta \lambda_{c}\left(z_{\mathrm{MF} 2}\right)=1.42 \mathrm{~nm}$, by turning the heating power on and off, see Figure $2 \mathrm{c} 1, \mathrm{c} 2$. This corresponds to a temperature difference $\Delta T\left(z_{\mathrm{MF} 2}\right)=110^{\circ} \mathrm{C}$. Taking into account the room temperature $22{ }^{\circ} \mathrm{C}$ during the experiment, we conclude that the maximum temperature of the MCF was $\sim 130{ }^{\circ} \mathrm{C}$, that is, above the water's boiling point. From the optical microscope images of the heated MCF and the transmission spectrum analysis described in Supporting Information, Section 2, we found no evidence of liquid water disappearing. The explanation of this phenomenon requires further investigation. It may be caused by the water flow, which leads to the nonuniform radial distribution of its temperature decreasing in the MCF center. Alternatively, water can remain in its liquid superheated state. ${ }^{52-54}$ The effect of WGM penetration into water is evident for $\mathrm{CW}$ variations with a smaller contrast and higher radial quantum numbers, that is, for that in the region between $\lambda=1551$ and $1552 \mathrm{~nm}$ in Figure $2 \mathrm{~b}$, which corresponds, approximately, to $F_{\mathrm{s}}=0.97$ and $F_{\mathrm{w}}=$
0.03. For CW variations with much smaller contrast, corresponding to WGMs with much deeper penetration into water (e.g., those between $\lambda=1546$ and $1548 \mathrm{~nm}), \Delta \lambda_{c}\left(z_{\mathrm{MF} 2}\right)$ is negative and, therefore, $F_{\mathrm{w}}>0.1$.

Now that the MCF has been characterized during initial heating, we measured the $\mathrm{CW}$ variation with time at a fixed position. We positioned MF1 at the axial coordinate of MF2, $z_{\mathrm{MF} 1}=z_{\mathrm{MF} 2}$ and measured the evolution of spectra, $P(\lambda, t)$, as a function of time, $t$, shown in Figure $2 c$, which was collected every $1 \mathrm{~min}$ during the $12 \mathrm{~h}$ of heating. The top axial eigenwavelengths of the temporary resonator are shown in Figure $2 \mathrm{c} 4$. It indicates the smooth growth in height of the microresonator. Yet, CWs with a smaller contrast and higher radial quantum numbers exhibit nonlinear and even nonmonotonic evolution (see Figure 2c3 and Supporting Information, Section 3). To explain the complex behavior of $\mathrm{CW}$ variations shown in these figures, we hypothesize that the processes leading to the positive $\mathrm{CW}$ variation saturate with time. In contrast, other processes, like the dissolution of silica, leading to the negative $\mathrm{CW}$ variation are continuously supported by the water flow and thus can evolve uniformly in time. The competition of these processes, which depend on the local temperature, time, and WGM distributions, results in the appearance of both positive and negative $\mathrm{CW}$ variations.

Finally, after completing $12 \mathrm{~h}$ of slow cooking, the unheated MCF is characterized with and then without water to determine the introduced irreversible nonuniformities. Figure $2 \mathrm{~d}$ shows the spectrogram of the water-filled MCF measured with resolution $\Delta z=10 \mu \mathrm{m}$. The permanent alterations induced after slow cooking are evident by comparing the original and final magnified CW profiles in Figures $2 \mathrm{a} 1$ and $\mathrm{d} 1$, respectively. Contrary to the behavior of axial eigen-wavelengths in Figure $2 \mathrm{~b} 2$ discussed above, the eigen-wavelengths in Figure $2 \mathrm{~d} 2$ are appropriately independent of the coordinate along the MCF. Figure 2e shows the spectrogram of the MCF after being emptied from water and left to dry for $12 \mathrm{~h}$, measured with $\Delta z=20 \mu \mathrm{m}$. Remarkably, the difference of CW profiles in Figure $2 \mathrm{~d} 2$,e 2 is imperceptible, while their relative total CW shift is small (see Figure 2d1,e1). This confirms that the evanescent penetration of the corresponding WGMs into water was small, ${ }^{47}$ justifying our earlier MCF temperature estimation. A comparison of Figure $2 b$ and $d$ shows that the induced $\mathrm{CW}$ variation nonlinearly grows with local temperature. Previous work has shown that microresonators with similar CW variation can be created at much higher temperatures by local annealing with a $\mathrm{CO}_{2}$ laser during 
several seconds. ${ }^{45,46}$ Here, the resonator introduced was created over significantly longer time and much lower temperature. We suggest that the physical mechanism of the slow cooking phenomenon can be explained by complex processes at the silica-water interface reviewed above, for example, hydration and hydrolysis, ${ }^{23-31}$ crack formation, ${ }^{30,31}$ and silica dissolution. ${ }^{33-37}$ In addition, momentum transfer between WGMs and water ${ }^{48}$ and the temperature gradient evident from Figure $2 \mathrm{~b}$ and eq 1 can induce the flow of water inside the MCF. ${ }^{55}$ Water flow replenishes fresh water at the silica-water interface, allowing the continuous reactions of hydrolysis and hydration. ${ }^{5}$

To compare the permanent alterations created over time, we measured spectrograms at different slow-cooking times shown in Figure 3. We heated the same MCF for total durations of 18, 40, and $57 \mathrm{~h}$ using MF2, which carried $63 \mathrm{~mW}$ of power. After each slow-cooking duration, the unheated MCF was characterized with the resolution $\Delta z=30 \mu \mathrm{m}$. The growth of the resonator height was approximately linear at the time interval between 18 and $57 \mathrm{~h}$ (similar to that shown in Figure $2 \mathrm{c} 4)$. Within the $1.3 \mathrm{pm}$ precision of the OSA used, we did not detect the degradation of the $Q$-factor during the cooking process. Analysis of the narrowest transmission power dips in the spectra measured at the maxima position, $z_{\mathrm{MF} 2}$ (insets of Figure 3) shows that the $Q$-factor of induced resonators exceeds $5 \times 10^{5}$.

Prospectively, we suggest that the programmed translation of the heating MF2 allows the creation of resonators with predetermined complex shapes. Indeed, the characteristic speed of resonator height growth following from Figure 3 gives $0.1 \mathrm{pm} / \mathrm{min}$. Assuming that $10 \mathrm{~s}$ is sufficient to translate the MF2 to the required axial coordinate and stabilize the MCF temperature distribution, we find the possible accuracy of the introduced $\mathrm{CW}$ variation can be as high as $0.02 \mathrm{pm} / 10$ s. A comparison of the $\mathrm{CW}$ variation as a function of axial coordinate $z$ for the spectrograms shown in Figure 3 can be linearly rescaled to each other. However, the dependence of the permanently induced CW variation on time is, in general, not linear. Future work will allow us to develop the method of the slow cooking of microresonators with predetermined shape required for microscale optical signal processing and ultraprecise microfluidic sensing. For example, this approach will allow us to reproducibly fabricate slow light delay lines and dispersion compensators. ${ }^{56,57}$ Complementary to our method, fine-tuning of the MCF with HF solution ${ }^{58,59}$ may be useful to introduce the required shift of the cutoff wavelengths.

We found that the axial dependence of induced $\mathrm{CW}$ variation $\Delta \lambda_{c}(z)$ is different for WGMs with different quantum numbers. This is explained by the fact that WGMs with higher radial quantum numbers penetrate deeper into water. It is also known that each polarization interacts with the surface of the optical fiber differently. ${ }^{60}$ We suggest that the solution of the inverse problem to determine the refractive index distribution inside the MCF from the experimental spectrograms (e.g., those shown in Figures 2 and 3) will gain a deeper insight into the physical and chemical processes near the silica-water interface. We hypothesize that these processes can be affected by optical WGMs in a more complex manner than straightforward heating of water and silica. Indeed, it is known that chemical reactions can be accelerated by light (see, e.g., refs 61 and 62). As another potential application, a few mms length of the slow cooked microresonator can be used as an ultraprecise spatiotemporal microfluidic sensor. ${ }^{63}$ In fact, due to different axial lengths of WGMs with different axial quantum numbers, their eigenwavelengths will shift differently in response to local microfluidic changes. The analysis of these shifts will allow us to determine the axial microfluidic distribution along the microresonator length and over its cross-section as a function of time.

It is of special interest to investigate the slow cooking process for materials other than silica and water. Different liquids (e.g., of varying $\mathrm{pH}$, aprotic, and colloidal) and capillary materials (soft glasses and polymers) can be used. While the replacement of water by other liquids is straightforward, it may be more challenging to consider microcapillaries fabricated from other materials, which have sufficiently low attenuation of light. In certain cases, a nanoscale coating of a silica microcapillary with a material of interest at the internal surface may solve this problem. Overall, future applications of the discovered slow cooking phenomenon range from fabrication of optical microresonators with unprecedented subpicometer precision to ultraprecise spatiotemporal microfluidic sensing. In addition, our demonstration reveals a new ultraprecise method of investigation of mechanical, physical, and chemical processes at the liquid-solid interfaces.

\section{ASSOCIATED CONTENT}

SI Supporting Information

The Supporting Information is available free of charge at https://pubs.acs.org/doi/10.1021/acsphotonics.0c01851.

Section 1: Spectrogram treatment; Section 2: Evidence of liquid water heating by evanescent WGMs; Section 3: Additional experiments (PDF)

\section{AUTHOR INFORMATION}

\section{Corresponding Author}

Gabriella Gardosi - Aston Institute of Photonic Technologies, Aston University, Birmingham B4 7ET, United Kingdom; 다. orcid.org/0000-0002-7655-2528; Email: gardosig@ aston.ac.uk

\section{Authors}

Brian J. Mangan - OFS Laboratories, Somerset, New Jersey 08873, United States

Gabe S. Puc - OFS Laboratories, Somerset, New Jersey 08873, United States

Michael Sumetsky - Aston Institute of Photonic Technologies, Aston University, Birmingham B4 7ET, United Kingdom

Complete contact information is available at: https://pubs.acs.org/10.1021/acsphotonics.0c01851

\section{Notes}

The authors declare no competing financial interest.

\section{ACKNOWLEDGMENTS}

The authors acknowledge funding from Engineering and Physical Sciences Research Council (EPSRC) under Grant EP/P006183/1 and Wolfson Foundation under Grant 22069.

\section{REFERENCES}

(1) Vansant, E. F., Van Der Voort, P., Vrancken, K. C., Eds. In Characterization and Chemical Modification of the Silica Surface; Elsevier: Amsterdam, 1995.

(2) Ehrlich, H.; Demadis, K. D.; Pokrovsky, O. S.; Koutsoukos, P. G. Modern Views on Desilicification: Biosilica and Abiotic Silica 
Dissolution in Natural and Artificial Environments. Chem. Rev. 2010, 110, 4656-4689.

(3) Sokolova, T. A. The Destruction of Quartz, Amorphous Silica Minerals, and Feldspars in Model Experiments and in Soils: Possible Mechanisms, Rates, and Diagnostics (the Analysis of Literature). Eurasian Soil Sci. 2013, 46 (1), 91-105.

(4) Wiederhorn, S. M.; Yi, F.; LaVan, D.; Richter, L. J.; Fett, T.; Hoffmann, M. J. Volume Expansion Caused by Water Penetration into Silica Glass. J. Am. Ceram. Soc. 2015, 98, 78-87.

(5) Lis, D.; Backus, E. H. G.; Hunger, J.; Parekh, S. H.; Bonn, M. Liquid flow along a solid surface reversibly alters interfacial chemistry. Science 2014, 344, 1138-1142.

(6) Schrader, A. M.; Monroe, J. I.; Sheil, R.; Dobbs, H. A.; Keller, T. J.; Li, Y.; Jain, S.; Shell, M. S.; Israelachvili, J. N.; Han, S. Surface chemical heterogeneity modulates silica surface hydration. Proc. Natl. Acad. Sci. U. S. A. 2018, 115, 2890-2895.

(7) Azam, M. S.; Cai, C.; Gibbs, J. M.; Tyrode, E.; Hore, D. K. Silica surface charge enhancement at elevated temperatures revealed by interfacial water signals. J. Am. Chem. Soc. 2020, 142, 669.

(8) Tréguer, P. J.; De La Rocha, C. L. The World Ocean Silica Cycle. Annu. Rev. Mar. Sci. 2013, 5, 477.

(9) Latour, I.; Miranda, R.; Blanco, A. Silica removal in industrial effluents with high silica content and low hardness. Environ. Sci. Pollut. Res. 2014, 21, 9832.

(10) Rondeau, V.; Jacqmin-Gadda, H.; Commenges, D.; Helmer, C.; Dartigues, J.-F. Aluminum and Silica in Drinking Water and the Risk of Alzheimer's Disease or Cognitive Decline: Findings From 15-Year Follow-up of the PAQUID Cohort. Am. J. Epidemiol. 2008, 169, 489496.

(11) Niibori, Y.; Kunita, M.; Tochiyama, O.; Chida, T. Dissolution Rates of Amorphous Silica in Highly Alkaline Solution. J. Nucl. Sci. Technol. 2000, 37, 349-357.

(12) Gong, Y.; Xu, J.; Buchanan, R. C. The Aqueous Corrosion of Nuclear Waste Glasses Revisited: Probing the Surface and Interfacial Phenomena. Corros. Sci. 2018, 143, 65-75.

(13) Crundwell, F. K. On the Mechanism of the Dissolution of Quartz and Silica in Aqueous Solutions. ACS Omega 2017, 2, 11161127.

(14) Tomozawa, M.; Kim, D.-L.; Agarwal, A.; Davis, K. M. Water Diffusion and Surface Structural Relaxation of Silica Glasses. J. NonCryst. Solids 2001, 288, 73-80.

(15) Ostroverkhov, V.; Waychunas, G. A.; Shen, Y. R. New information on water interfacial structure revealed by phase-sensitive surface spectroscopy. Phys. Rev. Lett. 2005, 94 (4), 046102.

(16) Dalstein, L.; Potapova, E.; Tyrode, E. The elusive silica/water interface: isolated silanols under water as revealed by vibrational sum frequency spectroscopy. Phys. Chem. Chem. Phys. 2017, 19, 1034310349.

(17) Nihonyanagi, S.; Yamaguchi, S.; Tahara, T. Ultrafast Dynamics at Water Interfaces Studied by Vibrational Sum Frequency Generation Spectroscopy. Chem. Rev. 2017, 117 (16), 10665-10693.

(18) Rosenberg, D. J.; Alayoglu, S.; Kostecki, R.; Ahmed, M. Synthesis of microporous silica nanoparticles to study water phase transitions by vibrational spectroscopy. Nanoscale $A d v .2019,1$ (12), 4878-4887.

(19) Isaienko, O.; Borguet, E. Hydrophobicity of hydroxylated amorphous fused silica surfaces. Langmuir 2013, 29, 7885-7895.

(20) Young, G. J.; Bursh, T. P. Immersion calorimetry studies of the interaction of water with silica surfaces. J. Colloid Sci. 1960, 15, 361369.

(21) Fubini, B.; Bolis, V.; Bailes, M.; Stone, F. S. The reactivity of oxides with water vapor. Solid State Ionics 1989, 32-33, 258-272.

(22) Robinson, R. S.; Yuce, H. H. Scanning Tunneling Microscopy of Optical Fiber Corrosion: Surface Roughness Contribution to ZeroStress Aging. J. Am. Ceram. Soc. 1991, 74 (4), 814-818.

(23) Rondinella, V.; Matthewson, M. J. Effect of chemical stripping on the strength and surface morphology of fused silica optical fiber. Proc. SPIE 1993, 2074, 52-58.
(24) Peng, C.; Song, S.; Fort, T. Study of hydration layers near a hydrophilic surface in water through AFM imaging. Surf. Interface Anal. 2006, 38, 975-980.

(25) Bunker, B. C. Molecular Mechanisms for Corrosion of Silica and Silicate Glasses. J. Non-Cryst. Solids 1994, 179, 300-308.

(26) Huang, Z.; Pickrell, G.; Wang, A. Penetration rate of water in sapphire and silica optical fibers at elevated temperature and pressure. Opt. Eng. 2004, 43 (6), 1272.

(27) Lezzi, P. J.; Tomozawa, M.; Blanchet, T. A. Evaluation of residual curvature in two-point bent glass fibers. J. Non-Cryst. Solids 2013, 364, 77.

(28) Lezzi, P. J.; Tomozawa, M. An Overview of the Strengthening of Glass Fibers by Surface Stress Relaxation. Int. J. Appl. Glass Sci. 2015, 6 (1), 34-44.

(29) Aaldenberg, E. M.; Aaldenberg, J. S.; Blanchet, T. A.; Tomozawa, M. Surface shear stress relaxation of silica glass. J. Am. Ceram. Soc. 2019, 102, 4573.

(30) Wiederhorn, S. M.; Fett, T.; Guin, J.-P.; Ciccotti, M. Griffith Cracks at the Nanoscale. Int. J. Appl. Glass Sci. 2013, 4, 76.

(31) Ciccotti, M. Stress-corrosion mechanisms in silicate glasses. J. Phys. D: Appl. Phys. 2009, 42, 214006.

(32) Mazer, J. J.; Walther, J. V. Dissolution kinetics of silica glass as a function of $\mathrm{pH}$ between 40 and $85^{\circ} \mathrm{C}$. J. Non-Cryst. Solids 1994, 170, 32.

(33) Fournier, R. O.; Row, J. J. The solubility of amorphous silica in water at high temperatures and high pressures. Am. Mineral. 1977, 62, 1052.

(34) Dove, P. M.; Han, N.; Wallace, A. F.; De Yoreo, J. J. Kinetics of amorphous silica dissolution and the paradox of the silica polymorphs. Proc. Natl. Acad. Sci. U. S. A. 2008, 105, 9903-9908.

(35) Wakabayashi, H.; Tomozawa, M. Diffusion of water into silica glass at low temperature. J. Am. Ceram. Soc. 1989, 72 (10), 18501855.

(36) Davis, K. M.; Tomozawa, M. Water diffusion into silica glass: structural changes in silica glass and their effect on water solubility and diffusivity. J. Non-Cryst. Solids 1995, 185 (3), 203-220.

(37) Ge, D.; Yang, L.; Li, Y.; Zhao, J. Hydrophobic and thermal insulation properties of silica aerogel/epoxy composite. J. Non-Cryst. Solids 2009, 355, 2610-2615.

(38) Vahala, K. J. Optical Microcavities. Nature 2003, 424, 839846.

(39) Fan, X.; White, I. M. Optofluidic Microsystems for Chemical and Biological Analysis. Nat. Photonics 2011, 5 (10), 591-597.

(40) Reynolds, T.; Riesen, N.; Meldrum, A.; Fan, X.; Hall, J. M. M.; Monro, T. M.; Francois, A. Fluorescent and Lasing Whispering Gallery Mode Microresonators for Sensing Applications. Laser Photonics Rev. 2017, 11, 1600265.

(41) Zhang, Y.; Zhou, T.; Han, B.; Zhang, A.; Zhao, Y. Optical BioChemical Sensors Based on Whispering Gallery Mode Resonators. Nanoscale 2018, 10, 13832-13856.

(42) Sumetsky, M. Nanophotonics of Optical Fibers. Nanophotonics 2013, 2 (5-6), 393-406.

(43) Strekalov, D. V.; Marquardt, C.; Matsko, A. B.; Schwefel, H. G. L.; Leuchs, G. Nonlinear and Quantum Optics with Whispering Gallery Resonators. J. Opt. 2016, 18 (12), 123002.

(44) Radulaski, M.; Zhang, J. L.; Tzeng, Y.; Lagoudakis, K. G.; Ishiwata, H.; Dory, C.; Fischer, K. A.; Kelaita, Y. A.; Sun, S.; Maurer, P. C.; Alassaad, K.; Ferro, G.; Shen, Z.; Melosh, N. A.; Chu, S.; Vučković, J. Nanodiamond Integration with Photonic Devices. Laser Photonics Rev. 2019, 13 (8), 1800316.

(45) Sumetsky, M. Theory of SNAP devices: basic equations and comparison with the experiment. Opt. Express 2012, 20, 2253722554.

(46) Sumetsky, M. Optical bottle microresonators. Prog. Quantum Electron. 2019, 64, 1-30.

(47) Hamidfar, T.; Tokmakov, K. V.; Mangan, B. J.; Windeler, R. S.; Dmitriev, A. V.; Vitullo, D. L. P.; Bianucci, P.; Sumetsky, M. Localization of light in an optical microcapillary induced by a droplet. Optica 2018, 5, 382-388. 
(48) Bar-David, D.; Maayani, S.; Martin, L. L.; Carmon, T. Cavity optofluidics: a $\mu$ droplet's whispering-gallery mode makes a $\mu$ vortex. Opt. Express 2018, 26, 19115-19122.

(49) Snyder, A.; Love, J. Optical Waveguide Theory; Springer, 1983. (50) Dmitriev, A.; Toropov, N.; Sumetsky, M. Transient reconfigurable subangstrom-precise photonic circuits at the optical fiber surface. 2015 IEEE Photonics Conference (IPC); IEEE: Reston, VA, 2015; pp 1-2.

(51) Vitullo, D. L. P.; Zaki, S.; Gardosi, G.; Mangan, B. J.; Windeler, R. S.; Brodsky, M.; Sumetsky, M. Tunable SNAP microresonators via internal ohmic heating. Opt. Lett. 2018, 43 (17), 4316.

(52) Yarin, L. P.; Mosyak, A.; Hetsroni, G. Fluid Flow, Heat Transfer and Boiling in Micro-Channels; Springer, 2009.

(53) Debenedetti, P. Metastable Liquids Concepts and Principles. Princeton Academic Press, 1996.

(54) Erné, B. H.; Snetsinger, P. Thermodynamics of Water Superheated in the Microwave Oven. J. Chem. Educ. 2000, 77, 1309.

(55) Karbalaei, A.; Kumar, R.; Cho, H. J. Thermocapillarity in microfluidics-a review. Micromachines 2016, 7, 13.

(56) Sumetsky, M. Delay of light in an optical bottle resonator with nanoscale radius variation: dispersionless, broadband, and low loss. Phys. Rev. Lett. 2013, 111, 163901.

(57) Sumetsky, M. Management of slow light dispersion and delay time characteristics with SNAP bottle resonators. CLEO: 2014; OSA: San Jose, CA, 2014; p STu3N.6.

(58) White, I. M.; Hanumegowda, N. M.; Fan, X. Subfemtomole Detection of Small Molecules with Microsphere Sensors. Opt. Lett. 2005, 30 (23), 3189.

(59) White, I. M.; Oveys, H.; Fan, X. Liquid-core optical ringresonator sensors. Opt. Lett. 2006, 31 (9), 1319.

(60) Gorodetsky, M. L.; Pryamikov, A. D.; Ilchenko, V. S. Rayleigh scattering in high-Q microspheres. J. Opt. Soc. Am. B 2000, 17 (6), 1051.

(61) Göstl, R.; Senf, A.; Hecht, S. Remote-controlling chemical reactions by light: Towards chemistry with high spatio-temporal resolution. Chem. Soc. Rev. 2014, 43, 1982.

(62) Houck, H. A.; Du Prez, F. E.; Barner-Kowollik, C. Controlling thermal reactivity with different colors of light. Nat. Commun. 2017, 8, 1869.

(63) Sumetsky, M. Slow light optofluidics: a proposal. Opt. Lett. 2014, 39, 5578. 